\title{
How to break the FoV versus thickness rule in MCAO
}

\author{
Roberto Ragazzoni, ${ }^{\text {a }}$, Carmelo Arcidiacono, Marco Dima, Piero Dri, Jacopo Farinato, Giorgia \\ Gentile, Demetrio Magrin, and Valentina Viotto \\ INAF-Astronomical Observatory of Padova
}

\begin{abstract}
Enlarging the searchable area for suitable reference stars in MCAO could allow to eliminate the need for lasers in ELTs. It has been previously claimed that this was not achievable because enlarging such a FoV would have diminished the thickness of the turbulence corrected by each DM. We show in this paper that there are ways to overcome this limitation.
\end{abstract}

\section{Introduction}

MCAO (Multi Conjugated Adaptive Optics) allows for compensation of a larger than the isoplanatic patch Field of View (FoV) with the usage of more than one reference star. In contrast with conventional Adaptive optics, now often referred to as Single Conjugated Adaptive Optics (SCAO) it employs more than a Deformable Mirror (DM) conjugated to different ranges along the line of sight of the telescope. Nominally three reference stars and two DMs are the minimum considered so far although recently it has been shown that MCAO can be effective with as low as just two reference stars. SCAO using natural Guide Stars (NGS) has been plagued by the problem of sky coverage. The introduction of MCAO enlarge potentially the FoV where to search for suitable NGS leading to a discussion about which sky coverage can be obtained with solely NGS. While for Ground Layer correction there is little doubt that enlarging the FoV lead to an effective augmentation of the number or the availability of NGSs, the same cannot be said for the DMs conjugated to the high altitude layers. In fact there is a geometrical limit where the pupils do no longer overlaps at a certain range for a given FoV. Surpassing such an angle there is no any gain into augmenting the FoV. This angle is of the order of a couple of arcmin for an $8 \mathrm{~m}$ telescope and scale to as much as half a degree for an $100 \mathrm{~m}$ telescope, adjusting around 10 to 12 arcmin for a $42 \mathrm{~m}$ telescope like the proposed E-ELT.

\section{The FoV versus thickness rule}

Provided that the aperture of the telescope is large enough there is apparently no limit into the FoV searchable for the NGSs other than practical ones (optical quality, vignetting, etc.). In reality the situation is more subtle as in MCAO the correction achieved at a certain DM optically conjugated at a given range is effective not just at that height but also at adjacent ones. In other words if an MCAO system, has a couple of DMs conjugated, say, at 0 and $9 \mathrm{~km}$ of range from the telescope entrance pupil, the high altitude layer DM is not demanded to correct just the turbulence occurring at the altitude of conjugation but also at others one. In principle, also the layer in the middle point between the two heights of conjugation (in this example at $4.5 \mathrm{~km}$ ) will be partially corrected by both the two DMs. How this correction is effective requires some more detailed study but it is easy to understand considering a Layer oriented MCAO system [1]. In the Layer oriented MCAO, in fact, assuming one wish to correct, say, a FoV of 2 arcmin, one will look for several stars within such a FoV. As two stars closer than the isoplanatic patch will, roughly, gives the same information, we assume hereafter that "filling" the FoV with reference stars means that there is a number of stars homogeneously distributed such that

\footnotetext{
a e-mail: roberto.ragazzoni@oapd.inaf.it
} the original work is properly cited. 
the average distance between two adjacent star is of the order of the isoplanatic patch. it can be shown that this is even too much stringent and that even less stars are needed (just because what does matter is the effective isoplanatic angle characteristic of a certain region of the turbulent atmosphere, but this discussion is irrelevant here). The LO-MCAO average all such measurements. Lets now take a layer into an intermediate position. Because it is not where one of the DMs, and hence of the wavefront sensors, is conjugated, the rays of the various reference stars will not be focused on a specific point on the layer (or, correspondingly, on the DM or on the detector of the pupil plane wavefront sensor) but it will be spread. Such a spreading depends upon the FoV, the larger with the large is such an angle. This makes the effect to average out any turbulence that is on a spatial scale smaller than the size of this patch. But this is exactly what is required if one want to make the better and uniform correction over the selected FoV. In fact, if any of this signal would not be averaged out, it will make the correction better into a given direction, and worst into others, still inside the selected FoV.

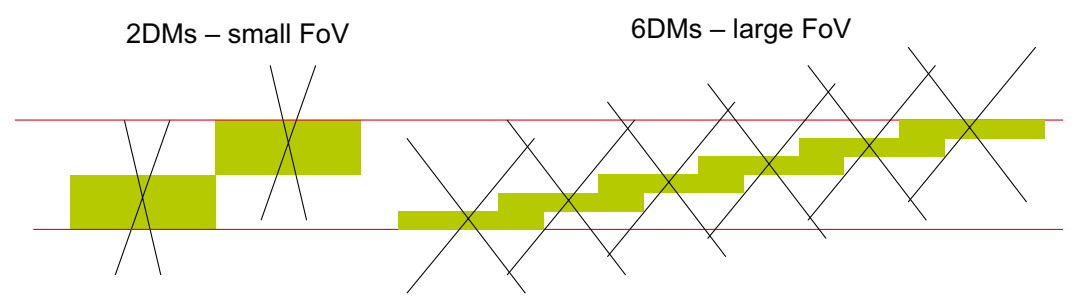

Fig. 1. To correct the whole column of turbulence for a given FoV, at a certain degree of correction, you need to introduce more than a single DM as soon as such an angle is larger than the isoplanatic angle. However, enlarging the FoV will degrade the thickness of the turbulence that you can compensate and you will need more DMs to achieve the same performances.

This means, however, that the degree of correction of a layer is limited for layers that are at a vertical distance from the ones where the DMs are conjugated. The turbulence will be smoothed out and the finest detail of the wavefront perturbation will get lost. Lets recall again that such a lost of information is deliberately on purpose. Any attempt to recover this information will result into inhomogeneous performances across the FoV. This also means, however, that the correction of the turbulence will be as much as possible, depending upon the finesse of the system (or the sampling of the pupil) for the selected layer and will degrade as much as the distance increase. One could make a practical limit to this. For example if the finesse is chosen equal to the Fried parameter $r_{0}$ one could choose a scale of three times such a parameter. One should always recall that the thickness of the turbulence that is so defined (the ones inside which the correction is achieved on a spatial scale, still following the numerical examples given above, up to three times the Fried parameter) is just indicative as the correction is always performed even at large distance, but only at much larger spatial scales (one should also recall that thanks to the Kolmogorov shape of the turbulence spectra such deterioration is not so huge, but we will not get into this in this framework). This has been sometime referred as the "Fov vs. thickness" rule of MCAO. If one, conventionally, is able to correct a FoV of. say, one arcmin with two DMs, each "compensating" a certain slab of the atmosphere, enlarging the FoV to a couple of arcmin will lead, unavoidably, to the shrinking of the thickness to a mere half. In the past this has been shown as a potential show-stopper for using NGSs for MCAO in ELTs.

\section{The experience of MAD}

In the meantime, thanks to the MAD experiment [2] Layer Oriented turned into reality. This has been accomplished through a wavefront sensor [3][4] with built-in eight positioning devices of corresponding stars-enlarger, suitable to cover the full FoV of 2 arcmin in diameter with certain limitation dictated by practical problems. In particular one can use up to four pairs of reference stars, each remaining into a quadrant covering a one arcmin in size each. Furthermore there is a minimum distance between each 
reference star to avoid mechanical collision between the various pick up systems. In practice, then the largest number of reference stars used has been limited to five. In some occasion we used as well, as in the "plain" MAD, just two reference stars as well. We focused our attention to the area of election for which the Layer-Oriented and all the subsequent techniques have been worked out, that is going to fainter reference stars. In fact most of the science produced in the six allocated scientific nights have been extragalactic, amounting probably alone to the largest fraction of MCAO science produced so far $[7][8][9][10]$.

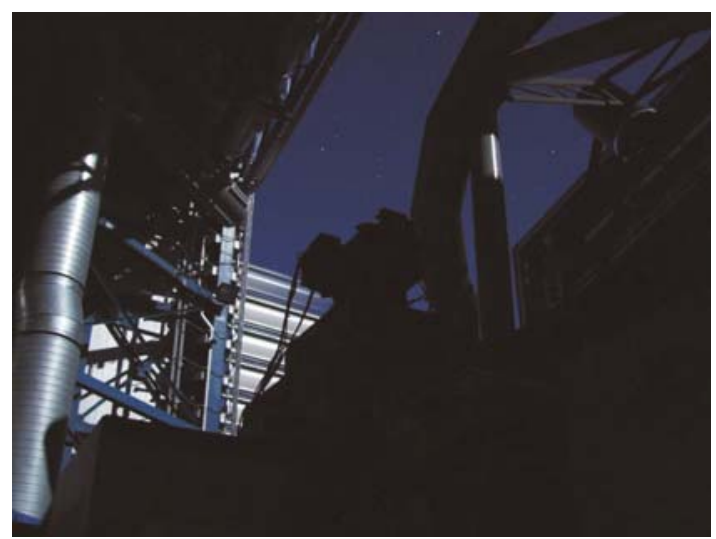

Fig. 2. The silhouette of the Layer Oriented Wavefront Sensor onboard MAD, at the Nasmyth foci of VLT during its observing run.

The LO-MAD performances turned out to be comparable to the conventional one in all the bright end cases and worked till much fainter magnitudes. There has not be any comparison in sky of the performances of the two approaches and, on the other hand, this would have been rather difficult to achieve giving the continuous variable conditions of the atmosphere. Laboratory tests has been limited to the sensitivity of the individual wavefront sensor or, in the LO case, for its use with a single reference. This confirmed the expected gain of the pyramid wavefront sensor and the magnitude gain obtained by comparison the curves of Strehl vs. apparent magnitude amount to a figure of the order of 1.5 magnitudes [5][6].

\section{Breaking the rules}

The simplest way to accomplish a larger FoV is just to... enlarge it. This, however, requires to introduce a much larger number of DMs in order to achieve a significant correction. Now, let us suppose that we build up a system with, say, five DMs. Each of the wavefront sensor will "see" reference stars in a much larger Field of View. Using a linear extrapolation say this could be achieved to a 10arcmin FoV. Now, the system is working (indifferent in Layer oriented or in a conventional mode) in perfectly closed loop fashion. Let us suppose to remove one of the DMs, but still to keep into a continuously updateable memory the information given to that DMs. Now one could easily compute which is the effect of that DM to each of the reference stars in terms of deterioration of the Wavefront. Just to be more precise we are not speaking here of the statistical deterioration but of the punctual evolution of the wavefront instant power instant, of course within the limitation of temporal and spatial sampling of the original, employing several DMs, MCAO system. Let us also assume that we are able to drive each WFs not around zero, or around a slightly different from zero position, but to a specific target identified by the computed effect on the wavefront from the removal of that DM. This of course implies a good enough linearity of the WF sensor. We will return to this point later but let us assume this for granted at the moment. Now we can make this "game" for all the DMs, in principle, and we could now de-project these to the correction necessary to achieve the full correction over just a normal FoV of a 
couple of arcmin with two DMs. Of course both the DMs have to be interested by the full 10 arcmin FoV. In this way we are not violating nothing and in fact the WF computer is "looking" apparently the same original system, that is an MCAO with five DMs. Of these, however, two are real and three are virtual. it is worthwhile to point out that these virtual DMs are continuously updated and are not used just for creating an interaction matrix.

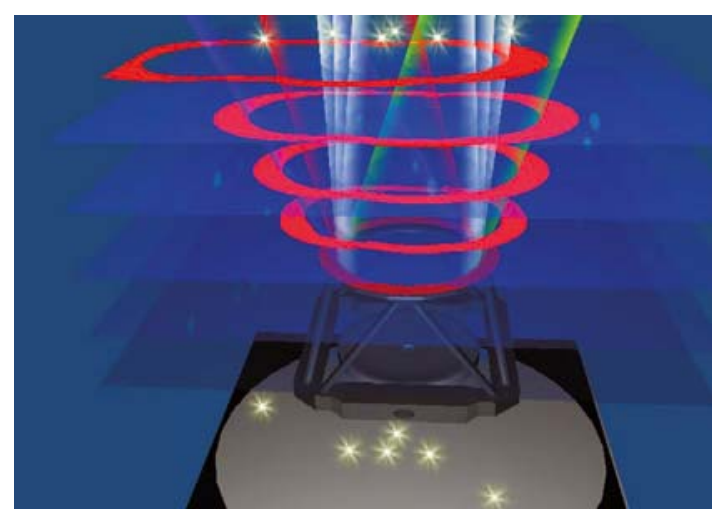

Fig. 3. Several layers, and several DMs, real or virtual, allow for searching for suitable Natural Guide Stars in a much larger Field of View.

Linearity of the WF sensor is a different issue. This can be worked out in a number of fashion. The most immediate and maybe the most effective is to replace the WFs with a small AO system each, maybe commanded through a MEMS, that actually work in closed loop, and to sense the small built-in DM with a fast sensor with high linearity (this can be an interferometric system as well as a conventional WF sensor with a deliberately deteriorated linearity, or being this illuminated by an artificial source, also a dedicated sensor). This unit would have the advantage of having the quality and performances of a WFs working ion coherent light (hence retaining, for example, all the gain of the pyramid one) without any further implication. A cheaper alternative could be to linearize an existing WF sensor for example applying a known tilt on the sensor at each measurement in order to estimate the degree of non linearity and to compensate them.

\section{Conclusion}

An MCAO system with solely NGS is preferable from the practical viewpoint for a number of reasons. First it eliminates in a single shot all the trouble deriving from the generation of the artificial stars (the laser and the launching system) and, second, it is much less invasive of the focal plane area than a LGS system based. It is questionable if the trouble of a system like the one proposed here requires so much linearity in the WF sensor to make it unfeasible. in fact one can easily see that this is exactly the same kind of problem of Multiple Object AO (MOAO). In this case the WF sensors and the corrected patches are uncorrelated. One can, in fact, see the proposed technique as an extension of the MOAO where the various patches merge together into a single and larger one located in the center of the FoV. It is also to point out that the requirements in terms of FoV imposed to the telescope to achieve a larger FoV are not more stringent than the one of collecting properly the LSGs rays that, because of the cone effect, arrive from a large angle. it is not by chance that the maximum FoV of the proposed E-ELT, 10 arcmin, fall exactly on the range of maximum angle where this technique have its maximum efficiency! Work is in progress to assess out the final sky coverage and to individuate various scenarios for the possible WF sensors for this kind of approach. 


\section{References}

1. Roberto Ragazzoni, Jacopo Farinato, Enrico Marchetti. SPIE 4007, (2000) 1076-1087.

2. Enrico Marchetti, Roland Brast, Bernard Delabre, Rob Donaldson, Enrico Fedrigo, Christoph Frank, Norbert Hubin, Johann Kolb, Jean-Louis Lizon, Massimiliano Marchesi, and 8 coauthors. SPIE 7015, (2008).

3. Elise Vernet-Viard, Carmelo Arcidiacono, Paolo Bagnara, Andrea Baruffolo, Emiliano Diolaiti, Jacopo Farinato, Matteo Lombini, Roberto Ragazzoni. Optical Engineering 44, (2005).

4. Roberto Ragazzoni, Emiliano Diolaiti, Elise Vernet, Jacopo Farinato, Enrico Marchetti, Carmelo Arcidiacono. The Publications of the Astronomical Society of the Pacific 117, (2005) 860-869.

5. Johann Kolb, Enrico Marchetti. MAD-TRE-0036, (2006)

6. C. Arcidiacono, M. Lombini, J. Farinato, R. Ragazzoni. Memorie della Società Astronomica Italiana 78, (2007) 708

7. R. Falomo, E. Pian, A. Treves, G. Giovannini, T. Venturi, A. Moretti, C. Arcidiacono, J. Farinato, R. Ragazzoni, E. Diolaiti, M. Lombini, F. Tavecchio, R. Brast, R. Donaldson, J. Kolb, E. Marchetti, S. Tordo.Astronomy and Astrophysics 501, (2009) 907-914.

8. A. Moretti, G. Piotto, C. Arcidiacono, A. P. Milone, R. Ragazzoni, R. Falomo, J. Farinato, L. R. Bedin, J. Anderson, A. Sarajedini, A. Baruffolo, E. Diolaiti, M. Lombini, R. Brast, R. Donaldson, J. Kolb, E. Marchetti, S. Tordo. Astronomy and Astrophysics 493, (2009) 539-546.

9. R. P. Mignani, R. Falomo, A. Moretti, A. Treves, R. Turolla, N. Sartore, S. Zane, C. Arcidiacono, M. Lombini, J. Farinato, A. Baruffolo, R. Ragazzoni, E. Marchetti. Astronomy and Astrophysics 488, (2008) 267-270

10. M. Gullieuszik, L. Greggio, E. V. Held, A. Moretti, C. Arcidiacono, P. Bagnara, A. Baruffolo, E. Diolaiti, R. Falomo, J. Farinato, M. Lombini, R. Ragazzoni, R. Brast, R. Donaldson, J. Kolb, E. Marchetti, S. Tordo. Astronomy and Astrophysics 483, (2008) L5-L8. 